\title{
Skin Disease Diagnosis System using Image Processing and Data Mining
}

\author{
R. S. Gound \\ Prof.: Dept. of IT \\ PCCOE, Nigdi, \\ Pune, India
}

\author{
Priyanka S. Gadre \\ Student: Dept. of IT \\ PCCOE, Nigdi \\ Pune, India
}

\author{
Jyoti B. Gaikwad \\ Student: Dept. of IT \\ PCCOE, Nigdi \\ Pune, India
}

\author{
Priyanka K. Wagh \\ Student: Dept. of IT \\ PCCOE, Nigdi \\ Pune, India
}

\begin{abstract}
Skin diseases are most common form of infections occurring in people of all ages. As the costs of dermatologists to monitor every patient is very high, there is a need for a computerized system to evaluate patient's risk of skin disease using images of their skin lesions. We will be constructing a diagnosis system based on the techniques of image processing and data mining. The procedure would be of great advantage to the dermatologists as a pre-screening system for early diagnosis in situations where the dermoscopes are not accessible. The proposed system will capture image through smart-phone camera. Preprocessing and segmentation will be performed on each image. Then Feature extraction is done on skin lesion Feature Extraction is very important for Predictive modeling applications. Feature extraction in image Processing is a method of capturing visual content of images for indexing and retrieval. Primitive image features can be either General features, such as extraction of color, texture and shape or Domain specific features. After feature extraction, feature classification can be done. In Feature Extraction, the system will compare the captured image with training dataset using image processing techniques and decides whether a skin suffers from diseases or not using decision tree. If there is disease, then the system will give medical advice through Android application
\end{abstract}

\section{Keywords}

Preprocessing, Segmentation, Feature Extraction, Feature Classification

\section{INTRODUCTION}

Skin is the largest organ present in the human body. Skin disease doesn't just affect the skin. It can have a huge impact on a person's day-to-day life, crush self- confidence, restrict their movement, and lead to depression and even ruin relationships. So it is needed to take skin disease seriously. [7] Skin diseases are very common among people of all age groups. In 2013, with prevalence rate of 10 percent, the population affected across India from skin disease was estimated at nearly 15.1 crore. It was estimated that at a CAGR of 12 percent about 18.8 crore people were likely to suffer from skin disease by 2015 [4]. Early diagnosis of melanoma is crucial and could lead to successful treatment. In real-life, a well-trained dermatologist could reach approximately $80 \%$ accuracy of diagnoses through visual inspection with specificity and sensitivity [5]. In this paper we propose a diagnosis system which will enable users to detect and recognize skin diseases with the help of image processing and data mining techniques and based on the result obtained in a shorter time period than the existing methods, we provide the user advises or treatments. We will be making use of java to perform the pre- processing and processing of the skin images of the users. This processing will be conducted on the different skin patterns and will be analyzed to obtain the results from which we can identify which skin disease the user is suffering.

\section{LITERATURE SERVEY}

Looking into the current situations of computerized skin disease diagnosis systems, there are few solutions available which are still under research developments. Certain limitations and drawbacks are identified in those hence this solution tries to overcome the existing problems with different approach. [4] [5] [1]

In the paper "Survey of Texture Based Feature Extraction for Skin Disease Detection" by Seema Kolkur, D.R. Kalbande, an approach of texture based feature extraction for detection of skin diseases has been presented to resolve issues. In statistical texture analysis, texture features are computed from the statistical distribution of observed combinations of intensities at specified positions relative to each other in the image. According to the number of intensity points (pixels) in each combination, statistics are classified into first-order, second-order and higher-order statistics. GLCM method is a way of extracting second order statistical texture features. Third and higher order textures consider the relationships among three or more pixels. These are theoretically possible but not commonly implemented due to calculation time and interpretation difficulty. In this paper, work on texture based features derived from GLCM matrix used for the detection of skin diseases is discussed and consolidated. Many researchers have used additional features along with texture based features to improve accuracy of classification. Most of the work is carried out detection of skin cancer but other diseases like psoriasis, warts, moles, eczema are also considered in some of the works. Classification of an image as having a disease or not is most carried by classifiers like neural networks and SVM. Most the research shows overall accuracy around or above $90 \%$. Top five features used in all this work are Contrast, Correlation, Energy, Entropy and homogeneity. Skin Disease Detection System based on these findings is proposed [4].

In the paper "An Intelligent Decision Support System for Skin Cancer Detection from Dermoscopic Images", the proposed system employs pre-processing such as dull razors and median filters to remove hair and other noise. Then, the images were segmented using a pixel limitation technique to separate lesions from image background. Feature extraction is subsequently conducted. The features extracted by the system reflect the well-known asymmetry, border irregularity, color variegation and diameter $(\mathrm{ABCD})$ of dermatology and the epiluminescence microscopy (ELM) criteria. They focus 
primarily on the size, shape, color and local parameters of lesions with some additional consideration of the lesion edges. The GA is also applied to identify the most discriminative feature subsets to improve classification accuracy. Evaluated with 100 images from the Edinburgh Research and Innovation (Dermofit) dataset, this work achieves an average accuracy of $92 \%$ and $84 \%$ for benign and malignant skin lesion classification [5].

In the paper "Detection of Malignant Skin Diseases Based on the Lesion Segmentation" by Jyothilakshmi K. K, Jeeva J. B, a novel technique to automatically detect the malignancy of skin diseases using conventional camera images is proposed. The procedure used would be of great advantage to the dermatologists as a pre-screening system for early diagnosis in situations where the dermoscopes are not accessible. This algorithm mainly aims at an early diagnosis of the malignant diseases since they can be cured if detected early. The proposed method works on color images by taking the HSV component and preprocessing was performed. A robust segmentation procedure is performed for the accurate detection of the lesion. For detection purpose, the morphological features like asymmetry, border irregularity, color variation and diameter are used. These extracted features help to identify the malignant lesions from the non-malignant ones [1].

\section{PROPOSED SYSTEM}

In this proposed system, we are considering a train of images that will be obtained from the user and preprocessing and segmentation will be performed on each image. Then feature extraction is done on each image to extract features that can be used to create classification model. With this classification model, system finally can predict the disease for a new image of a skin disease which will be obtained by the user through Android application. And based on this predicted disease, system will ask question from the user and based on answer, system will decide disease type. Finally, our system suggests medical treatment or the advice based on predicted skin disease result. In this system, we are taking into consideration three diseases viz. Eczema, Fungal infection, Urticaria.

Figure 1 shows system architecture, which shows the principal processes of the proposed system. In this section, we discuss the proposed methodologies in detail.

\subsection{Preprocessing}

Image pre-processing is an essential step of detection in order to remove noise such as hair clothing and other artifacts and enhance the quality of original image. The main purpose of this step is to improve the quality of skin image by removing unrelated and surplus parts in the back ground of image for further processing. Good selection of preprocessing techniques can greatly improve the accuracy of the system. The objective of the preprocessing stage can be achieved through three process stages of image enhancement, image restoration and hair removal.

\subsection{Segmentation}

Image segmentation is a technique to determine the shape and size of the border. It separates the object from its background based on different features extracted from the image. After removing the noise and hair from the lesion area, the lesion needs to be separated from the skin, and therefore the analysis for diagnosis is conducted purely using the necessary area. There are a lot of segmentation methods possible for this study



Fig. 1: System Architecture

\subsubsection{Thresholding}

This method determines the threshold and then the pixels are divided into groups based on that criterion. It includes bi-level and multi thresholding. Thresholding method includes Histogram and Adaptive thresholding

\subsubsection{Color-based segmentation algorithms}

Segmentation based on color discrimination. Include principle component transform/ spherical coordinate transform.

\subsubsection{Discontinuity-based segmentation}

Detection of lesion edges using active contours / radial search techniques / zero crossing of Laplacian of Gaussian (LoG). It covers Active contours, Radial search \& LoG

\subsubsection{Region-based segmentation}

It is a method of splitting the image into smaller components then merging sub images which are adjacent and similar in some sense. It includes Statistical region merging, multi scale region growing, and morphological flooding. It is based on 
the techniques such as Split and merge Statistical Region Merging Multi-Scale Morphological flooding

\subsubsection{Soft computing}

Soft computing techniques do the categorization of pixels using soft computing techniques. It includes Fuzzy Logic Neural Network, Optimization algorithms

\subsection{Feature Extraction}

A feature is a piece of information which is relevant for solving the computational task related to a certain application. Feature extraction is the process of extracting this information from an image. Following features can be extracted from the skin lesions.

\subsubsection{GLCM Features}

GLCM based features like Contrast, Correlation, Energy, Entropy and homogeneity.

\subsubsection{First-Order Histogram Features}

Features like Mean, Skewness, Energy computed form Firstorder histogram can be considered.

\subsubsection{Dermoscopic Features}

Dermoscopic features such as Asymmetry, Border, Color and Diameter (ABCD) of affected skin regions can be used.

\subsubsection{Color Features}

Mean color $(\mu \mathrm{c})$, standard deviation $(\sigma c)$ and skewness of color can be used.

\subsection{Feature Classification}

Selected features are used for the recognition and classification of benign and malignant lesions. A wide range of classifiers can be built and used for this purpose. Classifiers such as SVM, C4.5 can be used for this purpose.

\section{CONCLUSION}

In this study, an automated skin disease detection system is proposed which will help the medical society for the early detection of the skin diseases. This study is also highlighted to help the dermatologists in improving the diagnosis time and the accuracy of their intervention. As a future work, this method can also be tested on a large dataset consisting of various complexities to improve the efficiency of the algorithm. Also with the usage and the demand of the system we can expand the number of diseases which can be recognized by the system into considerable amount.

\section{REFERENCES}

[1] Jyothilakshmi K. K, Jeeva J. B, "Detection of Malignant Skin Diseases Based on the Lesion Segmentation", International Conference on Communication and Signal Processing, April 3-5, 2014, India

[2] Er. Shrinidhi Gindhi, Ansari Nausheen, Ansari Zoya, Shaikh Ruhin, "An Innovative Approach for Skin Disease Detection Using Image Processing and Data Mining", International Journal of Innovative Research in Computer and Communication Engineering, Vol. 5, Issue 4, April 2017

[3] A.A.L.C. Amarathunga, E.P.W.C. Ellawala, G.N. Abeysekara, C. R. J. Amalraj, "Expert System for Diagnosis of Skin Diseases", INTERNATIONAL JOURNAL OF SCIENTIFIC \& TECHNOLOGY RESEARCH VOLUME 4, ISSUE 01, JANUARY 2015

[4] Seema Kolkur, D.R. Kalbande, "Survey of Texture Based Feature Extraction for Skin Disease Detection", IEEE,2016.

[5] Teck Yan Tan, Li Zhang, Ming Jiang, “An Intelligent Decision Support System for Skin Cancer Detection from Dermoscopic Images", 2016 12th International Conference on Natural Computation, Fuzzy Systems and Knowledge Discovery (ICNC-FSKD)

[6] Uzma Jamil, Shehzad Khalid, "Valuable Pre-processing \& Segmentation Techniques Used in Automated Skin Lesion Detection Systems", 2015 17th UKSIM-AMSS International Conference on Modelling and Simulation.

[7] A.A.L.C. Amarathunga, E.P.W.C. Ellawala, G.N. Abeysekara, C. R. J. Amalraj, "Expert System for Diagnosis of Skin Diseases", INTERNATIONAL JOURNAL OF SCIENTIFIC \& TECHNOLOGY RESEARCH VOLUME 4, ISSUE 01, JANUARY 2015. 